\title{
Statistical Analysis of Temporal Variation of Heating Profiles Associated with Isolated Tropical Cold Cloud Systems by Using Satellite Observations
}

\author{
Keiji Imaoka ${ }^{1}$ and Kenji Nakamura ${ }^{2}$ \\ ${ }^{1}$ Graduate School of Environmental Studies, Nagoya University, Nagoya, and Earth Observation Research Center, \\ Japan Aerospace Exploration Agency, Tsukuba, Japan \\ ${ }^{2}$ Hydrospheric Atmospheric Research Center, Nagoya University, Nagoya, Japan
}

\begin{abstract}
Infrared observations from the Multi-functional Transport Satellite (MTSAT)-1R and latent heating profiles from the Tropical Rainfall Measuring Mission (TRMM) satellite are statistically analyzed to delineate the temporal variation of heating profiles associated with isolated cold cloud systems in the life cycle stages from the intermediate between initiation and mature to dissipating over tropical oceans. Clear temporal variations are confirmed for convective heating profiles, while those of the stratiform heating profiles are negligible. The resulting total profiles show that heating occurs at all levels throughout the lifetime of cold cloud systems, and the peak shifts from 5 to $8 \mathrm{~km}$ with elapsed time. Because that the area of stratiform rainfall in a system and its temporal variation are large, the total heating contribution released by a cold cloud system to the surrounding atmosphere during these life cycle stages is dominated by changes in the rainfall area and the ratio of the stratiform rainfall, rather than by changes in the shape of convective heating profiles.

(Citation: Imaoka, K. and K. Nakamura, 2013: Statistical analysis of heating profile changes associated with developing process of isolated tropical cold cloud systems by using satellite observations. SOLA, 9, 51-55, doi:10.2151/sola.2013-012.)
\end{abstract}

\section{Introduction}

The life cycles of cloud and precipitation systems have been investigated through many methodologies including ground-based radar observations, model simulations, and satellite observations. Although the basic concept of the life cycle is well understood, many aspects including its universality and quantitative nature remain unknown. Although statistical approaches are preferred, satellite observations can capture the representative view in global-scale dimensions. Many studies have employed geostationary infrared observations with cloud tracking methods for analyzing the cloud life cycle, such as those by Williams and Houze (1987) and Machado et al. (1998). To gain a wider range of information, recent studies have incorporated observations from lower orbiting satellites, particularly from the Tropical Rainfall Measuring Mission (TRMM) satellite, in addition to geostationary observations, such as those by Kondo et al. (2006), Futyan and Del Genio (2007), and Imaoka and Nakamura (2012). Moreover, a statistical approach for combining multiple low Earth-orbiting satellite observations was proposed by Masunaga (2012) to study the evolution of composite temperature and humidity fields on an hourly basis before and after the convection genesis. In recent years, satellite observations have been used to retrieve latent heating profiles from precipitating cloud systems (Tao et al. 2006). It is widely accepted that latent heat released by condensation plays an essential role in cloud evolution. The important role of the heating

Corresponding author: Keiji Imaoka, Earth Observation Research Center, Japan Aerospace Exploration Agency, 2-1-1 Sengen, Tsukuba, Ibaraki 3058505, Japan. E-mail: imaoka.keiji@jaxa.jp. (C)2013, the Meteorological Society of Japan. profiles associated with typical mesoscale convective systems (MCSs) in producing large-scale dynamics of the atmosphere (Hartmann et al. 1984; Schumacher et al. 2004) has also been recognized. As a ground-based effort, Frank and McBride (1989) compared composite analyses of tropical cloud clusters observed in the Australian Monsoon Experiment (AMEX) and the GARP Atlantic Tropical Experiment (GATE), focusing on the evolution of the life cycle and upon the vertical heating profiles, which were diagnosed from budget analyses of rawinsonde data. They found some differences in the life cycle of heating profiles between the systems in AMEX and GATE and hypothesized that the AMEX systems had stratiform rainfall from earlier life cycle stage than GATE systems. Although such ground-based experiments have been revealing the life cycle characteristics of heating profiles, large-scale systematic investigations such as by using satellite observations are not sufficient. This study describes the results of the analysis that combines geostationary infrared observations and heating profiles derived from TRMM data to show the statistical nature of the life cycle changes of heating profiles.

\section{Data and study method}

Satellite observations obtained from June 2006 to May 2010 over a rectangular region of $20^{\circ} \mathrm{S}-20^{\circ} \mathrm{N}$ and $90^{\circ} \mathrm{E}-180^{\circ} \mathrm{W}$, which includes the Maritime Continent and a part of the tropical western Pacific, as shown in Fig. 1, were used in this study. The study area is identical to that of Imaoka and Nakamura (2012). The two datasets used in this analysis include the gridded data of the IR1 $(11 \mu \mathrm{m})$ infrared channel of the Japanese Advanced Meteorological Imager on the Multifunctional Transport Satellite-1R (MTSAT-1R; Kigawa 2001) and the latent heat research product from the Precipitation Radar (PR) onboard TRMM based on the Spectral Latent Heating (SLH) algorithm (Shige et al. 2004, 2007). Non-grid (orbital) files of the version-02 product were used. To identify cold cloud systems, the same procedures as those used by Imaoka and Nakamura (2012) were applied, which include the minimum speed technique with a maximum velocity of $100 \mathrm{~km} \mathrm{~h}^{-1}$, an IR1 brightness temperature $(\mathrm{Tb})$ threshold of $235 \mathrm{~K}$, and a minimum cold cloud area corresponding to the area of a circle of radius $25 \mathrm{~km}$. The systems that did not exhibit merging and splitting were selected to obtain a clear statistical overview of the life cycle of heating profiles. Although this selection reduced the number of systems and their sizes, it still maintains the universality for discussing the typical cloud life cycle for small and short-lived systems (Kondo et al. 2006). After identifying the cold cloud systems over the target period and area, synchronized heating profiles with the cloud systems with temporal synchronization within $\pm 0.5 \mathrm{~h}$ were determined and used for further statistical analysis. The SLH algorithm used in the version- 02 heating profile product utilizes a lookup table produced from Tropical Ocean and Global Atmosphere Coupled Ocean-Atmosphere Response Experiment simulations. This lookup table relates the statistically optimum heating profile to the precipitation top height and rain rate at the melting layer for convective and deep stratiform rainfall, respectively (Shige et al. 2004). Therefore, despite a lack of PR sensitivity to the small ice-phase hydrometeors in the 

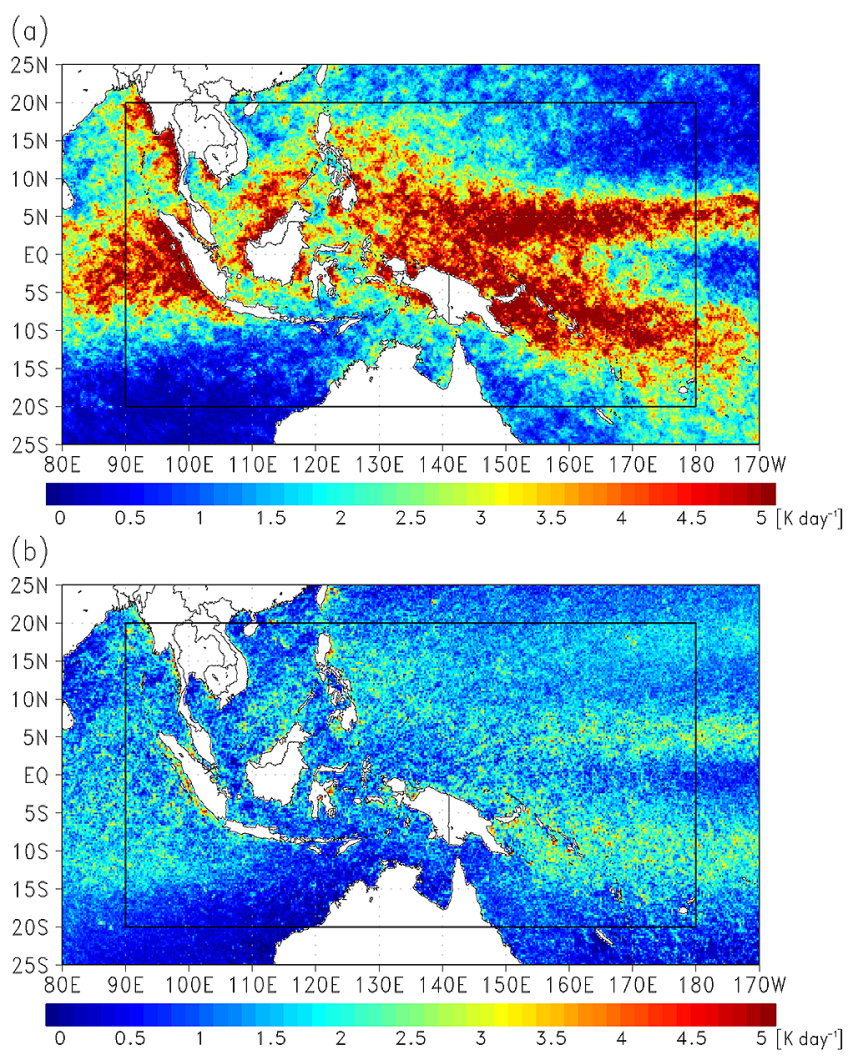

Fig. 1. Geographical distributions of four-year rain-unconditional average of $Q_{1 R}$ heating at (a) $7.5 \mathrm{~km}$ and (b) $2.0 \mathrm{~km}$ heights over tropical oceans. The black rectangular frame indicates the target area of this analysis.

anvil clouds, the algorithm can continuously track heating profiles throughout the cloud life cycle, with the exception of those in old convective cells without rainfall at the melting layer. In addition, universality of the lookup table was investigated by Shige et al. (2007) for the rainfall over oceans. After making several improvements, they reported reasonable performance of the SLH algorithm through consistency check by using true heating profiles produced by the cloud resolving model and comparison between the retrieved profiles from PR and those derived diagnostically from the South China Sea Monsoon Experiment sounding data. The latent heat research product includes the net latent heating by the phase change of water and $Q_{1}-Q_{R}$ (hereinafter $Q_{1 R}$ ) where $Q_{1}$ is the apparent heat source (Yanai et al. 1973), and $Q_{R}$ the cooling/ heating rate associated with radiative processes. In this analysis, we used the $Q_{1 R}$ estimate as a diabatic heating rate.

\section{Results and discussion}

In total, 306,664 cold cloud systems were identified by cloud tracking procedures described in the previous section for the target period and area, more than $90 \%$ of which fall within a lifetime range of $1-5 \mathrm{~h}$. The mean radius of the systems was less than $40 \mathrm{~km}$ for 5-h lifetime systems, which indicates comparable or smaller size than those of the typical MCSs reported in studies such as Houze $(1993,2004)$. Among these 1-5-h lifetime systems, 3105 systems were accompanied by TRMM synchronous observations. Figure 2 shows life cycle variations of the areas with PR rainfall within the cold cloud systems for 1-5-h lifetime systems. The convective rainfall area shown in Fig. 2 was derived by multiplying the total rainfall area with the ratio of the number of convective profiles to the total profiles. Although the stratiform rainfall areas in Fig. 2 actually included areas of all types of rainfall other than convective, contributions from the rainfall systems

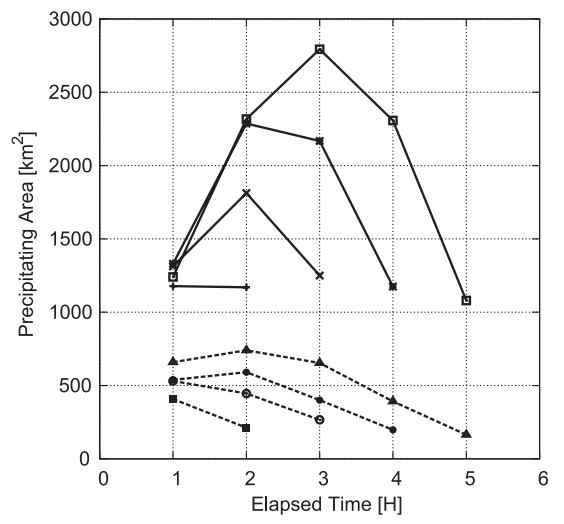

Fig. 2. Temporal variations of PR precipitating area within cold cloud systems for $2-5$-h lifetime systems over the ocean. Solid and dashed lines show the variations of areas with stratiform and convective rainfalls, respectively.

Table 1. Conditional rain rates (total and convective) and ratio of convective rainfall area for 2-h and 4-h lifetime systems.

\begin{tabular}{lccccc}
\hline & $\begin{array}{c}\text { Lifetime } \\
{[\mathrm{h}]}\end{array}$ & \multicolumn{5}{c}{ Elapsed Time [h] } \\
\cline { 3 - 6 } & 2 & 2.7 & 1.5 & - & - \\
\hline $\begin{array}{l}\text { Conditional rain rate (total) } \\
{\left[\mathrm{mm} \mathrm{h}^{-1}\right]}\end{array}$ & 4 & 3.5 & 2.4 & 1.7 & 1.2 \\
\hline $\begin{array}{l}\text { Conditional rain rate (convective) } \\
{\left[\mathrm{mm} \mathrm{h}^{-1}\right]}\end{array}$ & 2 & 7.0 & 5.1 & - & - \\
\hline Ratio of convective rainfall area & 2 & 26 & 15 & - & - \\
{$[\%]$} & 4 & 29 & 21 & 16 & 14 \\
\hline
\end{tabular}

such as warm rain were negligible because the present analysis can detect only cold cloud systems. Rain rates averaged over total rainfall area (rain-conditional average), convective rain-conditional average rain rates, and the ratio of convective rainfall area are shown in Table 1 for 2 -h and 4-h lifetime systems. As discussed by Imaoka and Nakamura (2012), all systems were accompanied by considerable areas of stratiform rainfall even in the beginning, indicating that the systems were in the intermediate stage, which is between initial and mature stages. Further details of these statistics can be found in Imaoka and Nakamura (2012).

Figures $3 \mathrm{a}$ and $3 \mathrm{~b}$ show the total, convective, and stratiform $Q_{1 R}$ heating profiles rain-conditionally averaged from June 2006 to May 2010 over the entire target area and over the identified cold cloud systems with $1-5$-h lifetimes. Averaging was performed over only the rainfall area (rain-conditional average). The convective heating profile in Fig. $3 \mathrm{a}$ indicates heating at all levels with two heating peaks at approximately 2 and $4.5 \mathrm{~km}$. In contrast, the stratiform heating profile in Fig. 3a shows cooling and heating at lower and upper altitudes, respectively, with the intersection with the y-axis at $5 \mathrm{~km}$. Because of averaging, the total profile in Fig. 3a shows heating at all levels with two maximums: one occurred at approximately $7.5 \mathrm{~km}$ mainly because of stratiform heating and the other at $2 \mathrm{~km}$ because of convective heating. The shape of the average total heating profile in Fig. 3a was apparently affected by the large area ratio of stratiform rainfall. On the other hand, the convective heating profile in Fig. $3 \mathrm{~b}$ shows a higher value of the peak at $4.5 \mathrm{~km}$ and no significant peak at $2 \mathrm{~km}$. This difference is easily understood because the profiles in Fig. 3a include all types of rainfall systems and those in Fig. 3b reflect only the characteristics of isolated cold cloud systems identified by the IR $\mathrm{Tb}$ threshold of $235 \mathrm{~K}$. The peak at $2 \mathrm{~km}$ in Fig. 3a possibly represents the characteristics of the cumulus congestus (Takayabu et al. 2010) and other shallow convective systems over tropical oceans. Figures 1a and $1 \mathrm{~b}$ show the geographical distribution of 

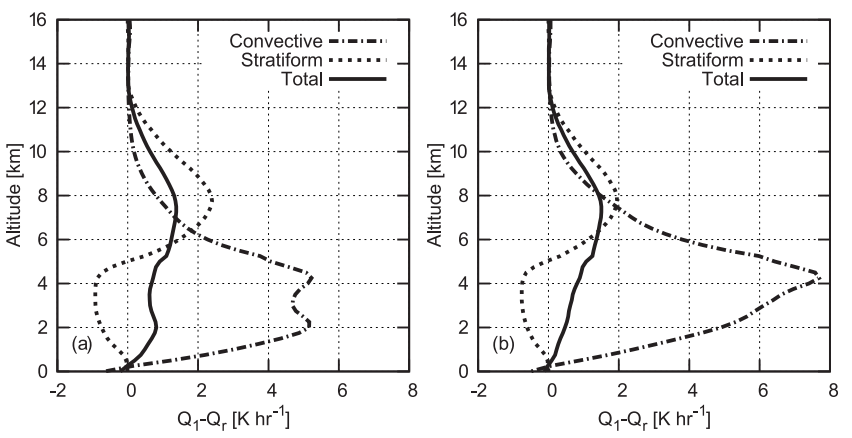

Fig. 3. Four year (June 2006-May 2010) average of total, convective, and stratiform $Q_{1 R}$ heating profiles over the (a) entire target areas and (b) identified cold cloud systems at 1-5-h lifetime.

the rain-unconditional average $Q_{1 R}$ heating rate at 7.5 and $2 \mathrm{~km}$ altitudes, respectively. As discussed by Takayabu et al. (2010), lower-level heating tends to follow the distribution of sea surface temperatures, such as the belt-like distribution from the Philippine Islands to Hawaii shown in Fig. 1b. Geographical distribution of the identified cold cloud systems moderately resembles to that of upper-level heating (not shown). Because the shapes of stratiform heating profiles, shown in Figs. 3a and 3b, are similar, the total heating profiles in the two figures do not differ significantly in amplitude and shape, with the exception of a small peak at $2 \mathrm{~km}$, shown in Fig. 3a. Therefore, regardless of the small number of occurrences and the small and short-lived characteristics of the isolated cold cloud systems observed in this study, the resulting total heating profile is similar in shape and amplitude as that over the entire tropical ocean region, which indicates that the results in this study may represent the features of heating profiles of tropical rainfall systems to some extent. The heating profiles shown in Fig. $3 \mathrm{~b}$ are consistent with previous observational findings such as those reported by Houze (1982) and Johnson and Young (1983) for MCSs, which include upper-level warming by condensation and deposition and lower-level cooling by evaporation of raindrops and ice particle melting.

Temporal variations of convective, stratiform, and total rain-conditional average $Q_{1 R}$ heating profiles are shown in Fig. 4 for the isolated cold cloud systems. Although these profiles were obtained for all systems with $1-5$-h lifetimes, only the results for the 2-h and 4-h lifetime systems are presented in the figure. For convective cases, the profiles indicate heating at all levels throughout the lifetime and clear temporal variations, as shown in Figs. $4 \mathrm{a}$ and $4 \mathrm{~b}$ for 2-h and 4-h lifetime systems, respectively. Maximum heating rates generally tended to appear at early hours of the lifetime and decreased with elapsed time. For the 4-h and 5-h lifetime systems, the heating rates at 2-h elapsed time are comparable to those observed in the beginning. The peak altitudes near $4.5 \mathrm{~km}$ remained constant throughout the lifetime. The maximum heating rate was larger (smaller) for the longer lifetime systems in the beginning (end), although the difference in the beginning is not prominent, as indicated in Figs. $4 \mathrm{a}$ and $4 \mathrm{~b}$. Compared with temporal variations of convective profiles, those in stratiform heating profiles were smaller, as shown in Figs. 4c and $4 \mathrm{~d}$. The heating rates in the upper troposphere slightly decreased with elapsed time, and the cooling rates at lower troposphere were nearly constant. The amplitudes and shapes of the profiles were fairly similar among 1-5-h lifetime systems, although the heating rates in the upper troposphere were rather larger in the beginning for the longer lifetime systems. The resulting total heating profiles indicate that all lifetime systems heat the atmosphere at all altitude levels throughout their lifetimes, as shown in Figs. 4e and 4f for 2-h and 4-h lifetime systems, respectively. A transition of the heating peak was observed from the middle altitude near $5 \mathrm{~km}$ to the upper troposphere near $8 \mathrm{~km}$. The total heating profiles and their temporal variations were considerably affected by the area ratio of convective and stratiform rainfall and its temporal chang-
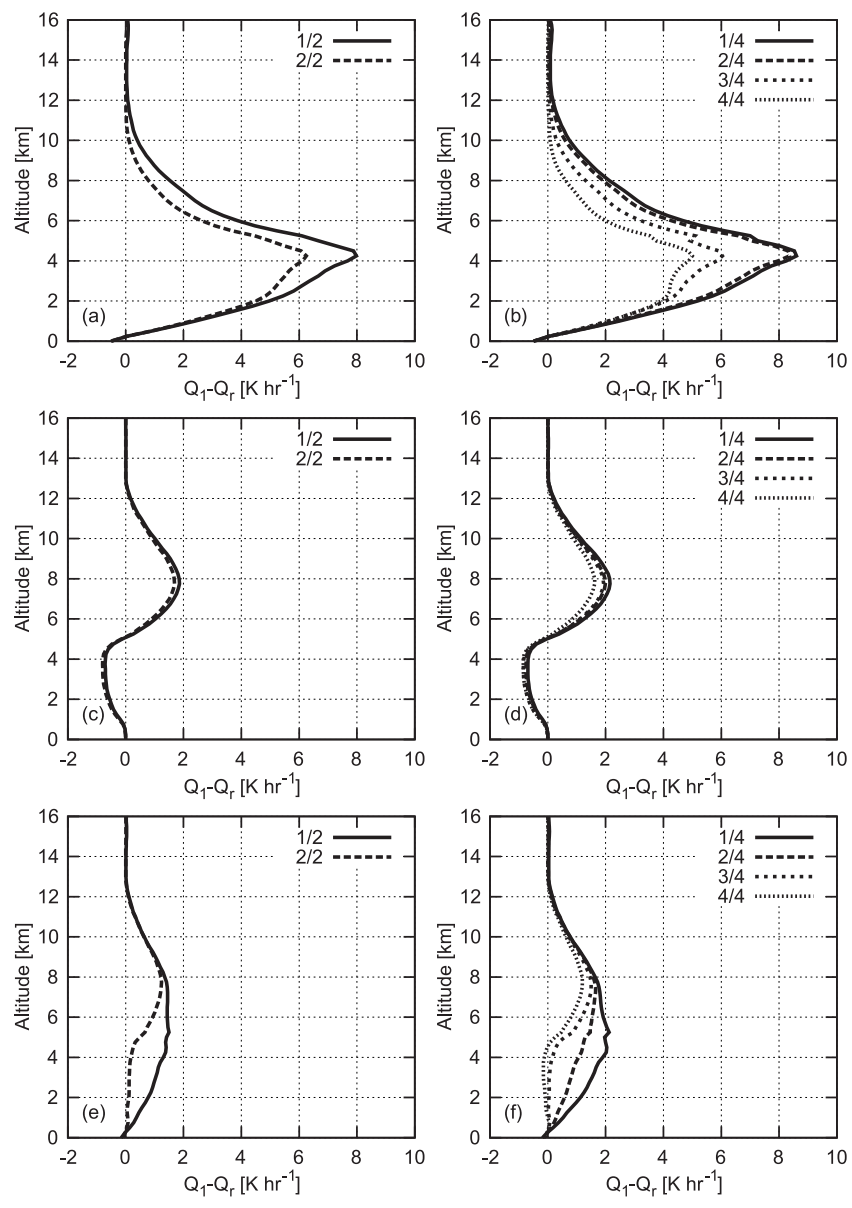

Fig. 4. Temporal variations of convective (top), stratiform (middle), and total (bottom) $Q_{1 R}$ heating profiles for cold cloud systems over oceans with lifetimes of $2 \mathrm{~h}$ (left column) and $4 \mathrm{~h}$ (right column). Different line types show elapsed time, which equals unity at the time of system formation.

es. The transition of the heating peak to the upper troposphere is attributed to the extension of a stratiform rainfall area in the later stages of the life cycle.

The total heating contribution released by a cold cloud system to the surrounding atmosphere can be expressed by the product of heating and rainfall area, as shown in Fig. 5. Because the areas of convective rainfall are larger for longer lifetime systems, as shown in Fig. 2, convective heating contributed by the 4-h lifetime system is considerably larger than that by the 2-h lifetime system in the early hours, as shown in Figs. 5a and 5b. The value is slightly smaller at the end of the lifetime because the area of the systems is comparable and the heating rate is smaller, as indicated in Figs. 2, 4a, and 4b. The differences in heating contribution between 2-h and 4-h lifetime systems are shown more clearly in Figs. $5 \mathrm{c}$ and $5 \mathrm{~d}$ for stratiform profiles. Because the extension of the stratiform rainfall area is large for the 4-h lifetime system, the heating (cooling) contribution at the upper (lower) troposphere is amplified and reaches its maximum (minimum) at 2-h elapsed time. By considering the rainfall area, the maximum convective heating and stratiform heating contributions shown in Figs. 5b and $5 \mathrm{~d}$, respectively, are nearly comparable in amplitude for the 4-h lifetime system. Therefore, the total heating contribution by the 4-h lifetime system, shown in Fig. 5f, indicates an amplified effect of stratiform heating contribution, whereas that by the 2-h lifetime system does not differ considerably from the profiles shown in Fig. 4e.

On the basis of these results, during the life cycle stages from the intermediate between initial and mature to dissipating, it is determined that the dominant factors for determining the temporal 

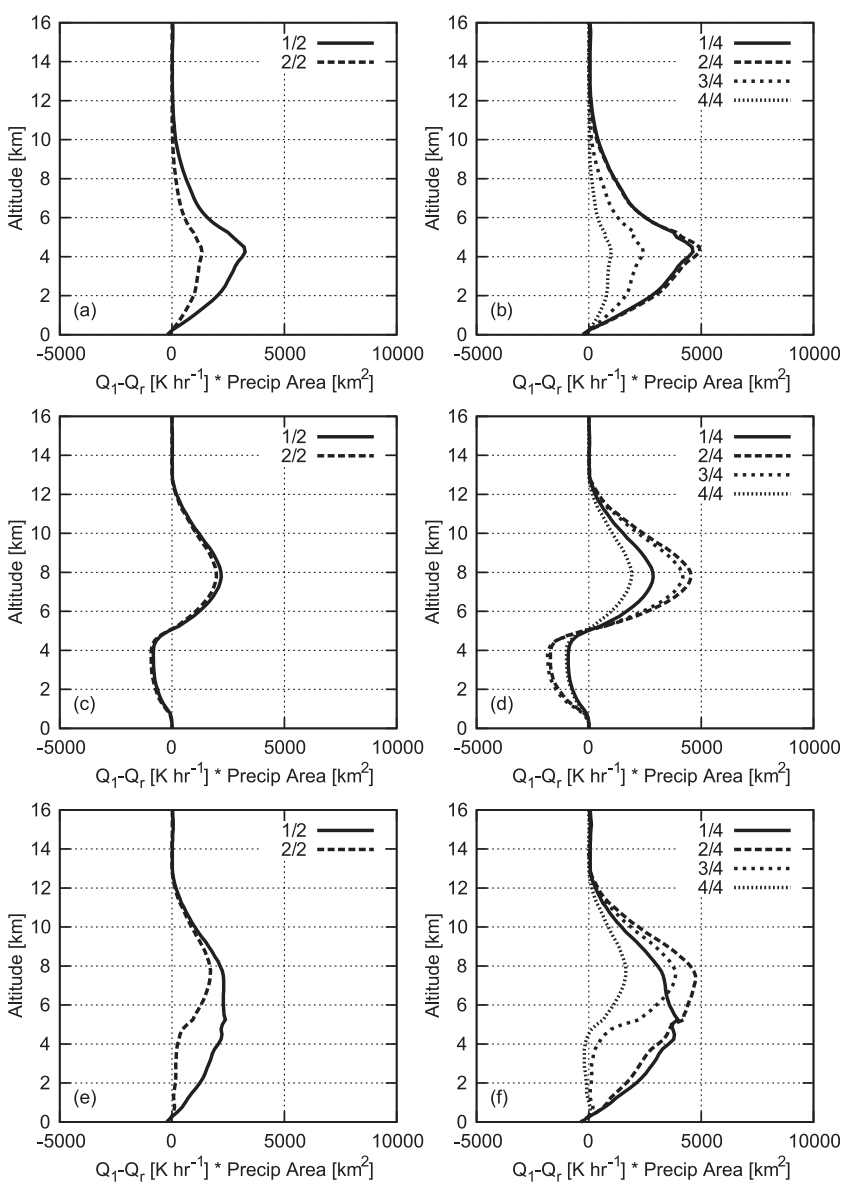

Fig. 5. Same as Fig. 4, but for with product of $Q_{1 R}$ heating and the rainfall area as abscissa.

variation of the total heating contribution from a cold cloud system are changes in the rainfall area and ratio of the stratiform rainfall, rather than changes in the shape of convective rainfall profiles. Houze $(1982,1989)$ demonstrated that the vertical structure of latent heat over tropical rainfall systems can be estimated by using the relative ratios of convective and stratiform rainfall. On the basis of this concept, Schumacher et al. (2004) estimated the tropical latent heating from the PR surface rainfall and stratiform rain fraction by assuming the typical heating profiles for convective and stratiform rainfall to show that the geographical and temporal variability in the stratiform rain fraction plays an important role in shaping the structure of the large-scale tropical circulation's response to precipitating cloud systems. Although a direct comparison cannot be made because of different research subjects, the present analysis does show similarities to the previous research. However, it should be noted again that the present analysis represents the cloud life cycle from the intermediate stage, between initial and mature stages, to dissipation, and does not capture the cloud life cycle at earlier stages. Recent studies, however, are leading to a high requirement for understanding the initiation of convective systems, and the role of shallow convections and their heating profiles in organizing cloud systems and in producing large-scale dynamics of the atmosphere. Therefore, future studies need to explore new techniques other than utilizing cold infrared Tbs to shed light on the understanding of the pre-initial and initial stage of the cloud life cycle. In addition, regional and environmental differences in heating profiles have been reported (Shige et al. 2007). For further investigation of the entire cloud life cycle over various regions, in which the shape of heating profiles may considerably vary, particularly in convective profiles, retrieval of accurate heating profile is essential. The SLH algorithm is suitable for this purpose; the algorithm is capable of retrieving realistic heating profiles throughout the cloud life cycle, as shown by Shige et al. (2004) for the evolution of a quasi-two-day oscillation. One weak point may be that this SLH algorithm cannot capture heating associated with old convective cells without rainfall at the melting level. Such issues could be further investigated by comparing the life cycle obtained by other instruments such as CloudSat, through which direct detection of anvil clouds is possible. Also, comparisons of the results obtained by using different heating profile products, such as derived by the convective-stratiform heating (CSH; Tao et al. 1993) and TRAIN (Grecu et al. 2009) algorithms, would be informative to understand the advantages and limitations of each algorithm. Comparison results can be found in previous papers such as Hagos et al. (2010). Although a time-series comparison was shown by Shige et al. (2004) for CSH and SLH algorithms, differences of characteristics in terms of their life cycle have not been fully investigated by previous studies.

\section{Conclusion}

Four years of satellite observations, including the $Q_{1 R}$ heating profiles from TRMM PR and the MTSAT-1R infrared data, were used to reveal the statistical nature of the temporal changes of rain-conditional average heating profiles associated with developing process of the isolated cold cloud systems over tropical oceans. The cold cloud systems identified in this study represent the life cycle phase from the intermediate stage, between the initial and mature stages, to dissipation, and do not include the systems at earlier stages. The similarity between the total heating profiles of the identified cold cloud systems with lifetimes of $1-5 \mathrm{~h}$ and those over the entire tropical ocean region indicates representativeness of this analysis to some extent, regardless of a small number of occurrences and the small and short-lived characteristics of isolated cold cloud systems. Through the present analysis, clear temporal variations were statistically confirmed, particularly for convective heating profiles, with heating observed at all levels throughout the lifetime, constant peak levels near $4.5 \mathrm{~km}$, and a decrease in heating rates with elapsed time. On the other hand, temporal variations were small in stratiform heating profiles, with heating observed at the upper troposphere and cooling at lower levels. These findings statistically confirmed life cycle characteristics of heating profiles, which have been investigated such as by ground-based observations, over the wide oceanic areas in the tropics. The resulting total heating profiles show heating at all levels during the lifetime and transition of the heating peak from approximately 5 to $8 \mathrm{~km}$ with elapsed time. Because the stratiform rainfall area and its life cycle changes are large, the total heating contribution released by a cold cloud system, which is expressed by the product of heating rate and rainfall area, is dominated by changes in the rainfall area and the ratio of the stratiform rainfall, rather than by changes in the shape of convective heating profiles in the life cycle stages from the intermediate between initial and mature to dissipating.

\section{Acknowledgments}

The authors wish to thank all members of the Laboratory of Satellite Meteorology, the Laboratory for Cloud and Precipitation Climatology (both of HyARC), and JAXA Earth Observation Research Center (EORC), for their helpful discussions and support. The gridded MTSAT-1R data were processed, archived, and provided by the Center for Environmental Remote Sensing (CEReS), Chiba University. The latent heat research product was provided by JAXA EORC. 


\section{References}

Frank, W. M., and J. L. McBride, 1989: The vertical distribution of heating in AMEX and GATE cloud clusters. J. Atmos. Sci., 46, 3464-3478.

Futyan, J. M., and A. D. Del Genio, 2007: Deep convective system evolution over Africa and the Tropical Atlantic. $J$. Climate, 20, 5041-5060.

Grecu, M., W. S. Olson, C.-L. Shie, T. S. L'Ecuyer, and W.-K. Tao, 2009: Combining satellite microwave radiometer and radar observations to estimate atmospheric heating profiles. $J$. Climate, 22, 6356-6376.

Hartmann, D. L., H. H. Hendon, and R. A. Houze Jr., 1984: Some implications of the mesoscale circulations in tropical cloud clusters for large-scale dynamics and climate. J. Atmos. Sci., 41, 113-121.

Houze, R. A., Jr., 1982: Cloud clusters and large-scale vertical motions in the tropics. J. Meteor. Soc. Japan, 60, 396-410.

Houze, R. A., Jr., 1989: Observed structure of mesoscale convective systems and implications for large-scale heating. Quart. J. Roy. Meteor. Soc., 115, 425-461.

Houze, R. A., Jr., 1993: Cloud Dynamics. Academic Press, 573 $\mathrm{pp}$.

Houze, R. A., Jr., 2004: Mesoscale convective systems. Rev. Geophys., 42, RG4003, doi:10.1029/2004RG000150.

Imaoka, K., and K. Nakamura, 2012: Statistical analysis of the life cycle of isolated tropical cold cloud systems using MTSAT-1R and TRMM data. Mon. Wea. Rev., 140, 35523572 .

Johnson, R. H., and G. S. Young, 1983: Heat and moisture budgets of tropical mesoscale anvil clouds. J. Atmos. Sci., 40, $2138-2147$.

Kigawa, S., 2001: Overview of MTSAT-1R Imager. Meteorological Satellite Center Technical Note, 39, 33-37.

Kondo, Y., A. Higuchi, and K. Nakamura, 2006: Small-scale cloud activity over the Maritime Continent and the Western Pacific as revealed by satellite data. Mon. Wea. Rev., 134, 1581-1599.
Machado, L. A. T., W. B. Rossow, R. L. Guedes, and A. W. Walker, 1998: Life cycle variations of mesoscale convective systems over the Americas. Mon. Wea. Rev., 126, 16301654.

Masunaga, H., 2012: A satellite study of the atmospheric forcing and response to moist convection over tropical and subtropical oceans. J. Atmos. Sci., 69, 150-167.

Schumacher, C., R. A. Houze Jr., and I. Kraucunas, 2004: The tropical dynamical response to latent heating estimates derived from the TRMM precipitation radar. J. Atmos. Sci., 61, 1341-1358.

Shige, S., Y. N. Takayabu, W.-K. Tao, and D. E. Johnson, 2004: Spectral retrieval of latent heating profiles from TRMM PR data. Part I: Development of a model-based algorithm. J. Appl. Meteor., 43, 1095-1113.

Shige, S., Y. N. Takayabu, W.-K. Tao, and C.-L. Shie, 2007: Spectral retrieval of latent heating profiles from TRMM PR data. Part II: Algorithm Improvement and heating estimates over tropical ocean regions. J. Appl. Meteor., 46, 1098-1124.

Takayabu, N. Y., S. Shige, W.-K. Tao, and N. Hirota, 2010: Shallow and deep latent heating modes over tropical oceans observed with TRMM PR spectral latent heating data. $J$. Climate, 23, 2030-2046.

Tao, W.-K., S. Lang, J. Simpson, and R. Adler, 1993: Retrieval algorithm for estimating the vertical profiles of latent heat release: Their applications for TRMM. J. Meteor. Soc. Japan, 71, 685-700.

Tao, W.-K., and co-authors, 2006: Retrieval of latent heating from TRMM measurements. Bull. Amer. Meteor. Soc., 87, 15551572 .

Williams, M., and R. A. Houze, 1987: Satellite-observed characteristics of winter monsoon cloud clusters. Mon. Wea. Rev., 115, 505-519.

Yanai, M., S. Esbensen, and J.-H. Chu, 1973: Determination of bulk properties of tropical cloud clusters from large-scale heat and moisture budgets. J. Atmos. Sci., 30, 611-627.

Manuscript received 15 December 2012, accepted 11 March 2013 SOLA: http://www.jstage.jst.go.jp/browse/sola 